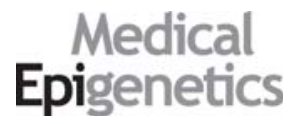

\title{
From Normal Development to Disease: The Biochemistry and Regulation of GLI2
}

\author{
Angela L. McCleary-Wheeler \\ Schulze Center for Novel Therapeutics, Mayo Clinic, Rochester, Minn., USA
}

\author{
Key Words \\ GLI2 · Transcription factor · Development · Cancer
}

\begin{abstract}
GLI2 is an oncogene encoding a unique transcription factor with both repressor and activator functions. Vitally important in development, it is also thought to be necessary for homeostasis of adult cells. However, deregulation of the GLI2 protein can result in detrimental effects to an organism, such as congenital defects or cancer. Historically deemed an activator and effector molecule of the Hedgehog signaling pathway, GLI2 has since been shown to be a critical effector of other signaling pathways, thus positioning itself as a potent mediator of signaling crosstalk. While GLI2 activity can be modulated by a variety of signaling influences, its regulation at the gene level is less understood. Indeed, gene mutations in GLI2 have been reported, but these generally led to developmental defects and are less commonly identified in tumors as being a cause of its deregulation. While the biological importance of GLI2 overexpression in a multitude of unrelated cancers has been well established, questions about the mechanisms leading to aberrant expression have remained largely unanswered. Furthering our understanding of both the transcriptional regulation of the GLI2 gene and the target genes regulated by GLI2 may identify novel therapeutic targets for cancer treatment.
\end{abstract}

\section{Organization and Transcriptional Activity of GLI2}

GLI2, along with the related GLI1 and GLI3, is a member of the GLI family of transcription factors belonging to the Glis superfamily. This Glis superfamily is characterized by a conserved DNA-binding domain consisting of five consecutive and symmetric zinc finger motifs in the pattern $\mathrm{X}_{3}$-Cys- $\mathrm{X}_{4}$-Cys- $\mathrm{X}_{12}$-His- $\mathrm{X}_{4}$-His- $\mathrm{X}_{3}$, where the $\mathrm{X}$ represents any amino acid [1]. A specific nine-nucleotide DNA sequence of $5^{\prime}$-GACCACCCA-3' is recognized by these GLI zinc fingers to mediate their binding to the DNA [1-3]. 
McCleary-Wheeler: From Normal Development to Disease: The Biochemistry and Regulation of GLI2

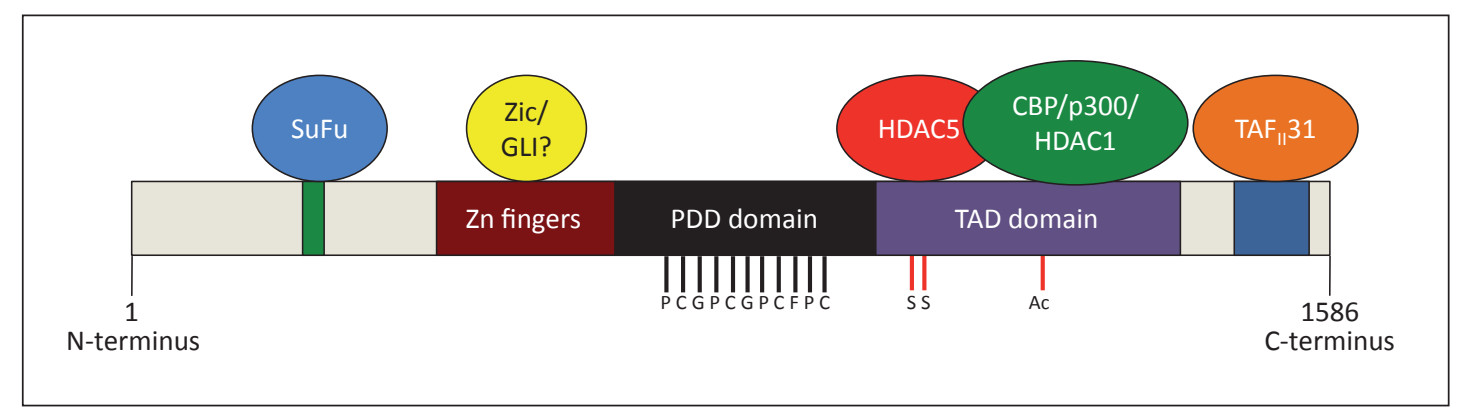

Fig. 1. Diagram of the modular organization of the GLI2 regulatory protein. The balloons located at the top indicate regulatory proteins that bind to GLI2. The black bars below the domain sequence indicate serine residues that are phosphorylated by PKA (P), CK (C), or GSK3 (G). The red bars indicate lysine residues that are sumoylated (S) or acetylated (Ac). These modifications are important for regulating GLI2 protein stability and activity. Zn fingers = Zinc fingers DNA-binding domain; Zic = zinc finger of the cerebellum-family transcription factors; PDD = processing determinant domain; TAD = transactivation domain.

\section{Biochemical Features of GLI2}

In addition to its zinc finger DNA-binding domain, the GLI2 protein also contains an $\mathrm{N}$-terminal repressor domain, a C-terminally located transcriptional activation domain, and domains identifying the nuclear localization and nuclear exportation signals (fig. 1) [4-8]. While some of these domains are better characterized than others, many are conserved within this family of transcription factors.

The suppressor of fused ( $\mathrm{SuFu}$ ) binding domain, nuclear localization signal, and nuclear export signal all play a role in determining where GLI2 localizes in the cell. As will be discussed in further detail later, the GLI proteins are highly regulated through Hedgehog (Hh) signaling. GLI2 can be left in an inactive state if $\mathrm{SuFu}$, a signaling protein downstream of the Hh pathway responsible for inhibiting GLI-mediated transcription, sequesters it in the cytoplasm. Evaluation of SuFu identified a C-terminal region that binds to the amino-terminal ends of the GLI proteins as well as a separate amino-terminal domain that binds specifically to the carboxyterminal end of GLI1 $[9,10]$. GLI1 was shown to contain a conserved SYGH motif that is specifically responsible for mediating the interaction with $\mathrm{SuFu}$, and glycine $\mathrm{e}^{122}$ and histidine ${ }^{123}$ of GLI1 are the critical amino acids involved [7]. What is important in the context of GLI2 is that GLI2 is the principal effector of this pathway, and its sequestration by SuFu plays a significant role in its activity. Alignment of the GLI1 and GLI2 protein sequences finds that GLI2 contains an identical SYGH motif and SuFu-binding site (amino acids 273-277) as GLI1 (amino acids 121-125) [McCleary-Wheeler, unpubl. data].

\section{GLI2 Interacts with Chromatin-Modifying Complexes}

Similar to other transcription factors, GLI2 modulates gene expression through the recruitment of coregulators to its target promoters. Part of this function may be mediated by the interaction of GLI2 with $\mathrm{TAF}_{\mathrm{II}} 31$, a factor that mediates transcriptional initiation, as GLI2 has a putative $\mathrm{TAF}_{\mathrm{II}} 31$ recognition motif [11, McCleary-Wheeler, unpubl. data]. However, GLI2 itself can be regulated at the level of the chromatin to alter its effects. Specifically, histone-modifying enzymes can act upon GLI2 to alter its ability to regulate the promoters of target genes. This may be through various mechanisms, such as acetylation, sumoylation, or through the interaction with repressive complexes (fig. 1).

GLI1, another member of the GLI family of transcription factors, can be acetylated on lysine 518, which inhibits its transcriptional activity, but it was speculated that this occurs on 
a respective lysine residue of GLI2, as histone deacetylase 1 (HDAC1) promoted an increase in GLI2 activity as well as GLI1 [12]. They further demonstrated that HDAC1-mediated deacetylation of GLI maintains a positive feedback loop as HDAC1 itself is a target of GLI [12]. However, the E3 ubiquitin ligase complex of Cullin3/REN antagonizes GLI activation by labeling HDAC1 for degradation [12]. In a more recent work, it was found that GLI2 was acetylated specifically by the histone acetyltransferase CREB-binding protein (CBP)/p300 on lysine 757, the amino acid correlating with lysine 518 of GLI1, resulting in decreased transcriptional activity of GLI2 and further supporting the previously described evidence acetylation at this location of GLI2 [13]. Mechanistically, GLI2 acetylation on lysine 757 prevented the association of GLI2 with chromatin at the level of its target promoters [13]. Additionally, mouse SuFu interacts with a component of the mSin 3 and HDAC complex called mSin3/ mSin3-associated polypeptide 18, or Sap18 [14]. This suppresses Gli (where Gli designates murine protein and GLI designates the human counterpart) activity, including that of Gli2 at the level of the Gli target gene promoter. Interestingly, acetylation of GLI2 by p300 may not only result in a reduction in GLI2 activity. The related protein, GLI3, is acetylated by p300 and the resulting modification turns on activator functions of GLI3 [5]. Additionally, Drosophila CBP recognizes and acetylates a specific sequence in the GLI homologue $\mathrm{Ci}$, thereby activating it [15]. The amino acid sequence recognized by CBP/p300 in Ci is conserved and is also found in GLI2, suggesting that modification by this complex may also activate GLI2 [6,16]. Thus, further description of the role of p300 in regulating GLI2 activity may be identified in future studies. While likely an important mechanism of GLI2 regulation, acetylation by histonemodifying enzymes is only one aspect of how GLI2 is regulated at the chromatin level.

GLI2 can be phosphorylated by protein kinase A (PKA). Phosphorylation of GLI2 by PKA results in its sumoylation at lysines 630 and 716 [17]. The addition of the small ubiquitin-like modifier (SUMO) group through sumoylation decreases GLI2 transcriptional activity through the recruitment of HDAC5 both in vitro and in vivo [17]. This prevents nuclear accumulation of GLI2 and a decrease in its transcriptional activation of its target genes. As such, this is a newly described mode of GLI2 regulation through the prevention of its nuclear localization and hence its association with chromatin.

One can appreciate from this review of GLI2 domains and posttranslational modifications of the protein that its activity can be manipulated through numerous mechanisms spanning several signaling pathways. Thus, the function of GLI2 can be affected through more than one signaling pathway. Additionally, the information presented thus far does not consider the regulation of GLI2 at the level of its own gene transcription. In fact, as will be highlighted later, this is one area of GLI2 regulation that has not been well described. Understanding the regulation of GLI2 at both the gene and protein levels is essential to fully understanding its role in biological processes. As detailed in the next sections, GLI2 is essential for normal development but at the same time, it is also implicated in disease, particularly for initiation and maintenance of some tumors.

\section{GLI2 Exerts Its Biological Impact in Development and Disease}

The biological effects modulated by GLI2 activity encompass a variety of functions at the individual cellular and whole organism levels. Target genes of GLI2 include important regulators of cell cycle progression, proliferation, and survival. Given the nature of these genes, GLI2 activity is important for developmental processes. However, with the implications for regulating such a gene expression program, GLI2 has been implicated in disease when its regulation goes awry (fig. 2). 
McCleary-Wheeler: From Normal Development to Disease: The Biochemistry and Regulation of GLI2

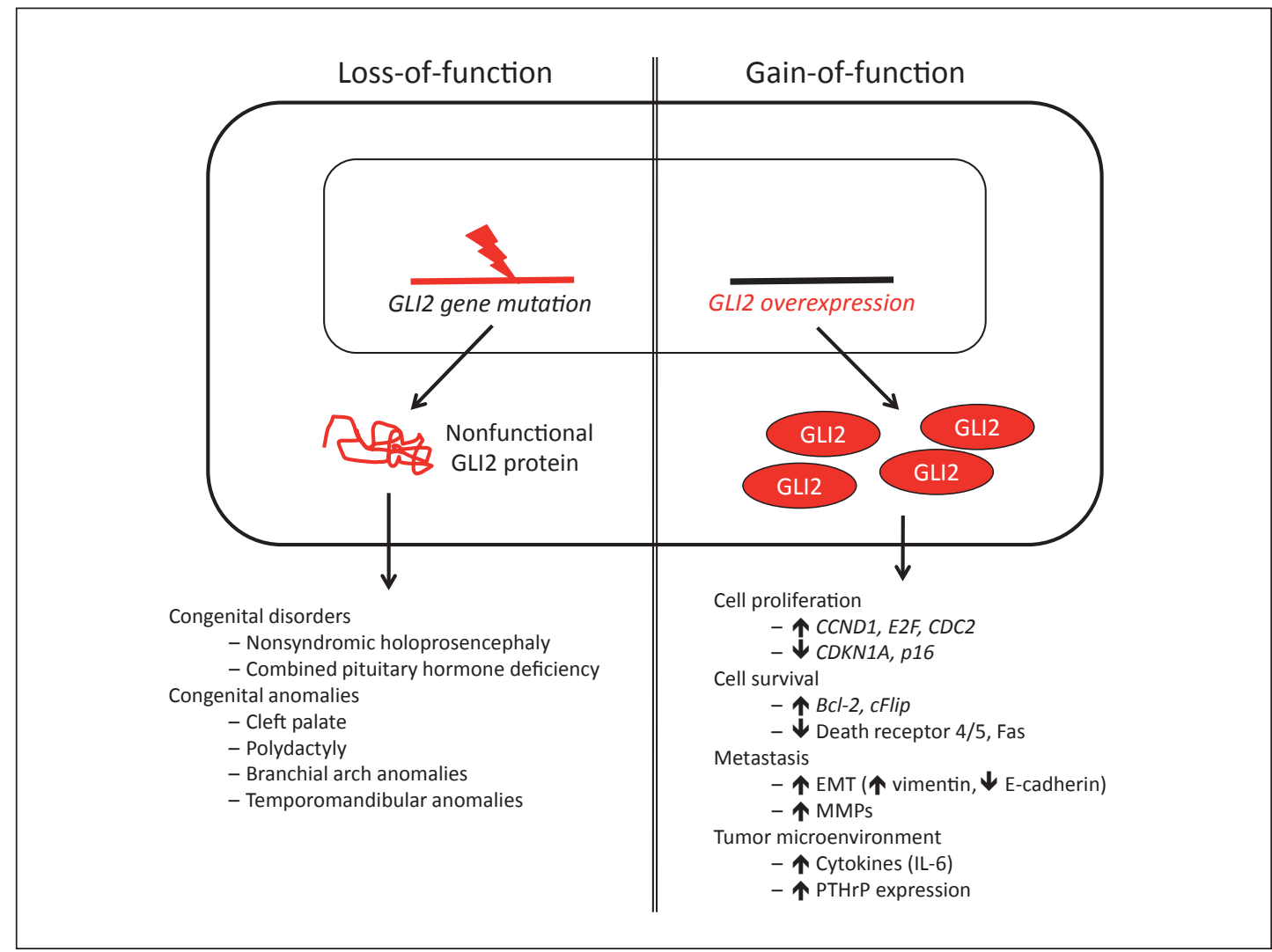

Fig. 2. Developmental defects and cancer are conditions that can develop secondary to abnormal GLI2 expression. On the left: GLI2 gene mutations can result in a nonfunctional protein that renders it unable to regulate patterning during development and results in congenital malformations. On the right: overexpression of GLI2 results in increased activity and potentiation of oncogenic functions.

\section{Role of GLI2 in Development}

GLI2 is an integral component of developmental programs. As an effector molecule of $\mathrm{Hh}$, a critical developmental pathway, much has been investigated regarding its role in development. This role can be quite complex, with its activity based on context-dependent signals. Because of this, much about what is known regarding GLI2 in developmental processes was garnered through the studies of loss of individual Hh effectors. This allowed for the developmental process regulated by GLI2 to be seen as either due to redundancy or specificity in cellular programming.

The function of the GLIs in development was initially determined through their homology with the Drosophila $\mathrm{Ci}$ and its role in patterning and tissue specification. Gli1, Gli2, and Gli3 are expressed in mouse embryos in regions that also highly express the Hh ligand [18]. The observation that mutations in the related GLI3 gene were responsible for a group of congenital malformations helped to prompt further investigations of the role of the GLIs, including GLI2, in the developmental process [11,19-21]. Multiple mouse mutants or knockouts have since been generated to study the effects on development. Mice with homozygous loss of functional Gli2 through the deletion of its zinc finger domains (Gli2 ${ }^{\mathrm{znf} / \mathrm{znf}}$ ) died in late embryonic stages (E18.5) or were stillborn, while heterozygous mice developed normally with no detectable abnormalities [22]. Specifically, expected Mendelian ratios were found earlier in development (E9.5) but decreased substantially at later time points. Thus, homozygous loss of Gli2 resulted 
in a lethal phenotype later in development [22]. Evaluation of the phenotypic abnormalities in these embryonic and newborn mice revealed flattening of the skull with lack of ossification, truncated mandibles with absent incisors, missing tympanic ring bones of the inner ear, and a range of severe cleft palate malformations [22]. The defects in palate development ranged from the lack of a presphenoid bone and maxillary and palatine shelves to only slightly abnormal shelves [22]. Further evaluations using the same Gli2 ${ }^{\text {znf/znf }}$ mice, severe foregut development defects were found, with stenosis of the esophagus and trachea and lung defects most notable [23]. These mice developed only one right lung lobe instead of the four that develop in wild-type mice, and the total lung size was smaller than either in wild-type or heterozygous mice [23]. Proliferation in the mutant lungs was lower later in development (by E14.5) than the normal lungs, suggesting a loss of cell growth in late development when compared to normal or heterozygous lungs [23]. Gli2-deficient mice also have defects in pituitary development that include partial loss of the anterior pituitary and complete loss of the posterior pituitary $[24,25]$. These pituitary defects were attributed to the loss of Bmp4 and Fgf8 expression in the ventral diencephalon in Gli2 mutants, indicating that these are target genes of Gli2 [25]. Perhaps most profound, and necessary for organism viability, are other central nervous system defects in mice lacking functional Gli2 where typically high levels of Sonic hedgehog (Shh), a ligand of Hh signaling, induce neural development. The floor plates in the midbrain, hindbrain, and spinal cord do not develop in homozygous Gli2 mutant mice, and the dopaminergic and serotonergic neurons that would normally flank the floor plate are missing in these animals $[26,27]$. However, they still develop the motor neurons lining the ventral midline of the neural tube, indicating that Gli2 is not needed in regions where Shh signaling is low $[26,27]$. These profound changes seen during development in mice deficient in functional Gli2 indicate its necessity for normal development of multiple organ systems. However, substantial redundancy in the Glis is also observed, and this makes it very challenging to truly isolate singular functions of GLI2. Moreover, the Gli2 knockout mice die late in embryonic development or at birth, making the characterization of cell-specific functions of Gli2 difficult.

While the changes noted with Gli2 deficiency alone in mice were found to be striking, it was also found that some of these changes overlapped with loss of Gli3. Homozygous loss of functional Gli3 through a $3^{\prime}$ deletion in the gene ( $\mathrm{Gli} 3^{\mathrm{xt}}{ }^{\mathrm{xt}}$ ) in mice resulted in abnormalities of the central nervous system, lung, and skeletal system that were similar to those in mice with loss of Gli2 [20]. When double mutant mice lacking Gli2 and Gli3 (Gli3 ${ }^{\mathrm{xt} / \mathrm{xtj}} / \mathrm{Gli}^{\mathrm{znf} / \mathrm{znf}}$ ) were generated, death occurred prior to complete skeletogenesis, and the mice had more severe defects than either single mutant $[22,23]$. Nevertheless, there was variation in the skeletal abnormalities seen, such as Gli2 or Gli3 loss separately affecting different areas of skull development and more limb abnormalities associated with Gli3 loss [22]. Thus, there was indication for both overlapping and specific functions of Gli2 and Gli3. This was also shown to be true for Gli1 and Gli2 as well. Mice expressing mutant Gli1 with lack of its zinc finger domains (Gli1 ${ }^{\text {nff/znf }}$ ) are viable and develop with no phenotypic abnormalities [24]. Double mutant mice with heterozygous loss of functional Gli2 on the Gli1 ${ }^{\text {znf/znf }}$ background had similar central nervous system and lung defects but milder than what was seen with loss of Gli2 alone [24]. Interestingly, when Gli1 was expressed at low levels in place of the endogenous Gli2 locus in mice, it was able to rescue the activity of Gli2 and the defects seen, including lethality [28]. However, when high levels of Gli1 were expressed over Gli2 this did cause lethality, as the replacement essentially became a Gli1 gain-of-function mutation [28]. Regardless, the conclusion drawn from these developmental studies confirmed that some of the functions of the Gli are redundant, particularly with Gli1 and Gli2, but this redundancy is not complete and expression of functional Gli2 is necessary for specific developmental programs. 
The specific programming dictated by GLI2 during development is generally confined to ventroposterior patterning of the mesodermal tissues. This is of particular significance in the development of parts of the central nervous system. Dorsoventral patterning of the neural tube is dictated in part through Shh signaling, where Shh is expressed in the ventral floor plate and notochord that become the neural tube and spinal cord [29]. Gli2 is required for the development of the ventral cell types in the spinal cord [28]. It is vital for the regulation of oligodendrocyte progenitor cells and differentiation and specification of the ventral aspect of the caudal neural tube [30, 31]. Gli2 can also function through fibroblastic growth factor signaling during neural development to mediate antero-posterior patterning by inducing the gene brachyury [32]. Spatial patterning of the midbrain and hindbrain in mouse models showed that Gli2 functions to induce neurons along the ventral aspect in a sequential medialto-lateral manner [33]. The role of Gli2 in neurogenesis was demonstrated by the observation that it is able to regulate the expression of neurogenic genes such as Ncam, Neurog1, and NeuroD [34]. While these aspects are important during development, GLI2 is necessary to help maintain a stem cell population in adult tissues. GLI2 maintains Nanog expression in adult neural stem cells, and Sox2 is also a target of GLI2 in neural stem cells [35, 36]. Using an embryonic stem cell model from the P19 embryonal carcinoma cell line, it was found that overexpression of GLI2 alone could induce the cells to undergo neuronal differentiation, and this was secondary to GLI2 directly targeting specific neurogenic factors like Ascl1/Mash1 [37]. In the cerebellum, GLI2 also serves as an activator and through stimulation of the proliferation of granule neuron precursor (GNP) cells $[38,39]$. The impact of these functions has the potential for disease consequences that will be discussed in the next section. Additionally, local gradients of the Shh ligand from the ventral midline cell compartments result in the formation of a dorsoventral axis forming the inner ear, with high quantities of activated Gli2 responsible for differentiation of the most ventral portion [40]. As highlighted by the numerous anomalies in mouse models of Gli2 loss during development, its role is not confined to the central nervous system.

GLI2 functions outside of the notochord and other central nervous system precursors during development to mediate development of other tissues. GLI2 plays a role in normal limb patterning, bone formation, and organogenesis during development. Gli2 plays a role in anterior-posterior patterning of the posterior limb in combination with Gli3 for proper limb development in the mouse [41]. Complete loss of Gli2 and Gli3 results in a lack of sclerotome formation by the somatic mesoderm in mice, causing skull and vertebral defects [42]. At least one copy of either Gli2 or Gli3 is needed to induce particular markers of sclerotome formation [42]. Hh signaling in mesenchymal cells and osteoblasts results in an induction of genes regulating osteoblast differentiation, such as alkaline phosphatase and osteocalcin, and initiation of calcification [43]. This was found to occur through Gli2, which directly increases expression of Runx2, a transcription factor necessary for osteoblastogenesis [43]. Gli2 can also regulate bone morphogenic protein 2 by directly binding to its promoter and stimulating its transcription [44]. It is necessary to mediate normal development of hair follicles in the epidermis of skin [45]. Furthermore, Gli2 is also the predominant mediator of the proliferation response to Shh in the developing lung by upregulating the expression of cyclin D1, D2, and E1 [46]. Gli2 functions in the developing immune system by mediating the expression of the transcription factor FoxA2 in double-negative thymocytes, thus preventing their differentiation to double-positive thymocytes [47]. Hh signaling is also known to be important in cardiac development, with loss of proper signaling resulting in alterations in heart tube formation, septal determination, and outflow tract impairments [48]. Gli2 helps to mediate this signaling by interacting with myocyte enhancer factor 2C. Gli2 and myocyte enhancer factor 2C are able to bind to each other's promoters to regulate their expression during cardiomyogenesis as well as form heterodimers with each other and synergize their activation of target genes 
important to this process [48]. Gli2 expression in the mouse epaxial somite is responsible for activation of the Myf5 epaxial muscle progenitor cells [49]. Furthermore, Gli2 is necessary for normal dorsoventral and mediolateral patterning of the epaxial muscle somite [49]. Ectopic expression of active Gli2 specifically in the pancreatic epithelial progenitor cells had no effect on pancreas development unless ablation of the primary cilia also occurred [50]. Under these circumstances, full $\mathrm{Hh}$ activation was permitted and this resulted in defective pancreas formation with a loss of both exocrine and endocrine cells, and the concomitant appearance of undifferentiated cells with pancreatic progenitor cell markers [50]. The culmination of these findings suggests that Hh signaling, and perhaps specifically GLI2, can alter not only pancreatic development but also function in the adult.

This detailed summary regarding the role of GLI2 in development gives way to the idea that it may play an important role in disease. One may note in particular that the fundamental role of GLI2 is that of a terminal effector of a potent developmental signaling pathway. Consequently, defects in the expression or function of GLI2 can result in diseases that range from congenital malformations to cancer.

\section{GLI2 and Disease: Congenital Malformations and Cancer}

As detailed thus far, GLI2 is a transcription factor vitally important to controlling genes responsible for cell growth, survival and differentiation. Emphasis has been put into the description of the role of GLI2 in development because this is when GLI2 is necessary, and its loss has profound effects in normal development. GLI2 is similar to components involved with other developmental pathways in that its expression and activity in differentiated cells and adult tissues tends to decrease. With these observations, one would speculate that the deregulation of GLI2 would lead to disease. Indeed, studies investigating the pathogenesis of numerous human diseases have identified GLI2 as a key culprit. While a spectrum of disorders and diseases has been described, there are generally two categories of disease mediated by GLI2 loss of function or deregulation: congenital disease and cancer. In general, a loss of function during development is responsible for congenital malformations while a gain of function in adult cells can lead to tumorigenesis (fig. 2).

Given its role in development, alterations in GLI2 function could result in severe defects in organogenesis. In fact, the observed malformations resulting in mice expressing mutant Gli proteins were similar to those observed for some human malformations. A key example is that of holoprosencephaly (HPE), a condition of incomplete or failed forebrain separation early during gestation. It has been found that loss-of-function mutations in human GLI2 that render an inactive protein product is associated with a type of HPE characterized by abnormal anterior pituitary formation and hypopituitarism with an inappropriately divided forebrain [51]. Additionally, nonsense and frameshift mutations in GLI2 have been identified in families with subsequent loss of the activator domain [52]. The patients affected had loss of a normal anterior pituitary and an associated loss of growth hormone, lack of pituitary hormones, and various facial defects [52]. Additionally, given the role GLI2 in regulating limb bud patterning, variable polydactyly was also identified [52]. Various other reported mutations in GLI2 have resulted in a spectrum of phenotypes that ranged from cleft palate, polydactyly, branchial arch abnormalities, various degrees of HPE, and temporomandibular joint anomalies [53]. These examples highlight the need for GLI2 to be tightly regulated and function normally to ensure proper fetal development. It is likely that other defects in GLI2 expression during development could occur, but as noted in murine models of Gli2 loss, many do not survive after birth. In contrast to these described congenital malformations and the syndromes associated with them, deregulation of GLI2 leading to increased activity can occur in adult cells and tissues. 
As part of a developmental signaling pathway, GLI2 is typically less active in adult cells, with the exception of maintenance of some adult stem cells, such as neural stem cells. However, when GLI2 becomes upregulated and is no longer subject to normal control processes, proliferative diseases, such as cancer, can arise. In fact, its close relative GLI1 is an oncogene that was originally identified as an amplified and highly expressed gene in human glioma [54]. Hence, GLI is an acronym for glioma-associated oncogene. The status of GLI2 as an oncogene has also been well established, as outlined in the following paragraphs. Increased expression of GLI2 has been identified in a variety of tumor types, such as basal cell carcinoma, diffuse large B-cell lymphoma, endometrial carcinoma, osteosarcoma, melanoma, colon cancer, prostate cancer, transitional cell carcinoma, pancreatic adenocarcinoma, some types of breast cancer, and ovarian cancer [55-65]. The following sections will discuss the various roles that GLI2 plays in cancer development and progression including cell proliferation and cell cycle progression, cell survival, metastasis, and regulation of the tumor microenvironment.

Several reports have revealed that GLI2 functions as an oncogene due, in part, to alterations in cell proliferation. Transgenic overexpression of Gli2 in basal keratinocytes using the keratin 5 promoter resulted in the development of basal cell carcinomas in mice [55]. Further studies using an inducibly expressed Gli2 transgene in mouse keratinocytes showed that Gli2 was necessary for tumorigenesis and maintenance, as when the gene was turned off, the basal cell carcinomas regressed [66]. As a marker of proliferation, Ki67 was increased when Gli2 was turned on, but it decreased when Gli2 was turned off [66]. Studies investigating GLI2 in prostate carcinoma found that its expression was increased in these tumors, but it decreased following initiation of hormonal therapy only to increase again when the tumors became resistant [61]. Specific knockdown of GLI2 with siRNA in prostate tumor cells has shown that loss of GLI2 results in a decrease in proliferation through the concomitant decrease in the expression of cell cycle progression genes such as cyclin D1, p21, and PKC- $\eta$ [61]. In contrast, overexpression of GLI2 in prostate cancer cell lines increased cell proliferation, and evaluation by flow cytometry found significantly more cells in the S-phase [67]. While these studies show the importance of GLI2 in promoting the proliferative capacity of a cell, the molecular mechanisms were not defined. However, some studies have shown more mechanistically the ways in which GLI2 promotes this behavior.

Some investigations have successfully identified genes directly targeted by GLI2 and defined mechanisms of GLI2-induced cellular proliferation. One study investigated proliferation-associated genes that may be targeted by GLI2 through expression of a constitutively active form of GLI2 in HaCaT cells and gene array profiling followed by qPCR validation of some of the results. E2F1, CDC45L, and CDC2 are upregulated by GLI2 along with CCNA2, $C C N B 1$, and $C K S 1 B$ [68]. Additionally, genes involved in cell cycle inhibition, such as CDKN1A, are downregulated by GLI2 [68]. In their study, Regl et al. [69] also used array profiling to identify alterations in genes when GLI2 was overexpressed in keratinocytes. They found that the genes that were most upregulated included Ptch, E2F1, CDC45L, PCNA, CDC2, and CCND1, while some genes such as $M Y C$ and $C D K N 1 A$ were significantly downregulated upon overexpression of GLI2 [69]. Thus, the results of these two studies correlated well. Upon further evaluation of the biological impact of these gene alterations, keratinocytes overexpressing GLI2 overcame their normal contact inhibition and continued to proliferate as demonstrated by increased 5-bromo-2'-deoxyuridine incorporation and increased numbers of cells in the S-phase [69]. Often, the increased proliferative effects mediated by increased GLI2 expression are assumed to be due to an increase in the positive cell cycle regulators that it regulates, but in these profiling studies, cell cycle inhibitors were implicated as targets of downregulation by GLI2. This was further elaborated upon by work performed by Bishop et al. [70] that revealed $p 16$, which is known to function as a cell cycle inhibitor, as a direct target of GLI2. In HMEC cells transfected with siRNA against GLI2, cell proliferation was suppressed while $p 16$ 
expression increased. A GLI-binding site was identified in the $p 16$ promoter; reporter and ChIP assays showed that GLI2 could bind and induce the activity of this promoter [70]. Cyclopamine, an inhibitor of GLI2 activation, decreased proliferation, increased $p 16$ expression, and caused eventual cell senescence [70]. Thus, this example not only highlights the ability of GLI2 to regulate the expression of a negative cell cycle regulator, but further, it shows an inhibitory function of GLI2 through promoter binding and transcriptional repression of a target gene. While exerting its activity on cell cycle regulators is critical to its function as an oncogene, GLI2 can regulate other targets that can precipitate its oncogenic behavior.

As a complementary function to cell cycle regulation, GLI2 also regulates cell survival factors, thus preventing cells from undergoing apoptosis. One gene that is often critical in the prevention of cell death is the antiapoptotic gene $\mathrm{Bcl}-2$. When transgenic mice overexpressing Gli2 in keratinocytes developed basal cell carcinomas, one of the genes subsequently found overexpressed was $\mathrm{Bcl}-2$ [55]. While $\mathrm{Bcl}-2$ gene expression was not specifically evaluated, in another inducible mouse model of Gli2 overexpression in basal cell carcinoma, lower caspase activity suggested a decrease in apoptosis of the cancer cells when Gli2 was turned on and an increase in apoptosis with regression of tumors when Gli2 was turned off [66]. Similar findings were shown in the case of human prostate carcinoma, hepatic carcinoma, and colon carcinoma cell lines where GLI2 function or expression was inhibited [60, 61, 71]. With many strong associations between GLI2 expression and Bcl-2, researchers specifically analyzed if Bcl-2 was a direct target of GLI2. Using promoter analysis, reporter assays and electrophoretic mobility shift assays (EMSA), it was found that Bcl-2 had GLI-binding sites and GLI2 binding to a specific site within the Bcl-2 promoter was necessary for the activation of Bcl-2 [69]. While Bcl-2 is an antiapoptotic protein, other antiapoptotic genes were also identified to be potential targets of GLI2.

The pro-survival gene $c$ Flip functions as an inhibitor of caspase-8. It was initially found that increased expression of GLI2 corresponded with an increase in expression of cFlip [72]. Promoter analysis revealed the presence of two consensus GLI-binding sequences. Reporter assays and EMSA found that GLI2 was capable of activating and binding to the cFlip promoter [72]. Knockdown GLI2 decreased cFlip expression, and apoptosis occurred in the cells [72]. Hence, the cFlip gene is another example of a direct GLI2 target gene. While few genes have been studied thoroughly to identify them as direct transcriptional targets of GLI2, further associations between GLI2 expression and the expression of other cell survival factors exist. In pancreatic cancer cells and pancreatic cancer stem cells, inhibition of GLI2 activity induced not only Bcl-2 expression, as expected based on previous reports, but also increased the expression of Fas ligand, death receptor 4, and death receptor 5 [73]. While enhanced cell proliferation combined with increased resistance to cell death are key to initiation and maintenance of tumorigenesis, other alterations in cell programming give way to more aggressive and invasive phenotypes that highlight metastatic properties.

Partly defining a malignant tumor is its ability to invade surrounding tissues and structures and the capacity to generate metastatic lesions. Understanding the modes in which tumors demonstrate such malignant features is necessary to help improve treatments and outcomes in many cases. Not surprisingly, GLI2 also has a role in this process. As in the previous descriptions, many associative type observations have been made regarding the expression levels of GLI2 in correlation with expression levels of markers of a metastatic phenotype. In transitional cell carcinoma cell lines, overexpression of GLI2 increases the invasive nature of these cells in Matrigel invasion assays [62]. GLI2 expression had been known to be increased in hepatocellular carcinoma when compared to nearby normal liver, but it was also determined that this increase in expression correlated with intrahepatic metastasis and vascular invasion [74]. Lending further explanation to this finding, immunohistochemistry of these tumors revealed that an increase in GLI2 expression also correlated 
with a decrease in the expression of E-cadherin and an increase in vimentin [74]. This association is observed in multiple tumor types. In some cases, mechanisms through which GLI2 can result in these changes have been identified.

Malignant melanoma is well documented to be an aggressive cancer with a high rate and extent of metastasis. In a study by Alexaki et al. [59], the importance of GLI2 to the invasive phenotype was studied in malignant melanoma. It was found that in melanoma cell lines having a high expression of GLI2, there was also a decrease in the expression of E-cadherin [59]. This corresponded to a more invasive phenotype of these cells when they were grown in Matrigel. An orthotopic mouse model also showed that the melanoma cells with a high expression of GLI2 formed bone metastasis. Additionally, activation of the TGF $\beta$ pathway revealed similar findings [59]. Finally, loss of GLI2 gene expression also resulted in a decrease in the secretion of matrix metalloproteinases, MMP-2 and MMP-9 [59]. While these results suggest that GLI2 is able to repress the expression of E-cadherin and hence may mediate an epithelial-to-mesenchymal transition (EMT) of cells, one possible mechanism explaining this repression was only recently elucidated. GLI2 was found to have a complex set of mechanisms for regulating the E-cadherin promoter. First, it was found that GLI2 is actually able to promote transcriptional activation of ZEB1, a protein known to promote an EMT phenotype [75]. Evaluation of the E-cadherin promoter found that Kruppel-like factor binding sites were present, and both GLI2 and ZEB1 were bound to these sites simultaneously [75]. GLI2 and ZEB1 were able to form nuclear complexes, as determined by proximity ligation assays [75]. As will be detailed later, the TGF $\beta$ pathway is known to induce GLI2 transcription as well as EMT. The activation of TGF $\beta$ in melanoma cells increased the GLI2/ZEB1 complexes found in the nucleus [75]. Likely, much more will be identified regarding the role of GLI2 in the regulation of EMT-related genes in the future. Despite the different mechanisms by which GLI2 functions as an oncogene in tumor cells, it also acts outside of the tumor cells in the surrounding tumor microenvironment.

The tumor microenvironment consists of the cells and matrix surrounding the tumor cells themselves. The cellular components of the tumor microenvironment include fibroblasts, immune cells, endothelial cells, and even neural cells. While these cells comprising the tumor microenvironment themselves are not neoplastic, they interact with each other and with the tumor cells. Complex and evolving interactions between the cells of the tumor and the cells of the surrounding microenvironment often result in a reprogramming of the cells in the microenvironment to positively support the tumor. Plenty of evidence exists proving that GLI2 has a role in modulating the tumor microenvironment through a variety of mechanisms.

The cells in the tumor microenvironment, including immune cells and stromal cells, may be manipulated by GLI2. The immune system is critical for recognizing developing tumor cells and destroying them before they have the ability to continue to proliferate and expand. At the same time, tumors secrete cytokines to impair or alter the response of specific immune cells. GLI2 acts in such a manner in different tumor types. In one case, GLI2 was found to regulate the expression of TGF $\beta 1$ in human CD4+ cells. Researchers have shown the presence of GLI2binding sites in the TGF $\beta 1$ promoter in CD4+ cells [76]. Reporter and ChIP assays showed that GLI2 was able to bind to two of these sites to promote transcriptional activation of TGF $\beta 1$ [76]. Since regulatory $\mathrm{T}$ cells are associated with a poor prognosis in many tumors, understanding their regulation and function for the purposes of aiding in cancer therapy may be important. Elsawa et al. [77] showed that induction of GLI2 in bone marrow stromal cells induces the expression and subsequent secretion of IL-6. They found that IL-6 is a direct target of GLI2 through reporter assays and ChIP analysis. Furthermore, the resultant production and secretion of IL- 6 into the microenvironment impacts malignant B cells. IL- 6 binds to and activates the IL- 6 receptors on malignant B cells that then leads to IgM secretion 
[77]. These findings identified a novel pathway through which malignant cells and cells of the microenvironment engage each other. Manipulation of the surrounding stromal cells and matrix is essential to those tumors that metastasize. This is especially true in the case of tumors that metastasize to bone. These tumors erode bone as they grow, and this is often the result of osteolysis caused by the parathyroid hormone-related peptide (PTHrP) that is secreted by the tumor cells. Using breast cancer cell lines that express high levels of PTHrP, GLI2 activity was decreased by expressing a repressor form of GLI2, and the result was a substantial decrease in PTHrP mRNA expression [78]. When GLI2 was overexpressed in breast cancer cell lines having a low expression of GLI2 and no or little PTHrP expression, an increased activity of a PTHrP reporter was seen [78]. To evaluate this relationship in vivo, cells stably expressing GLI2 were injected into nude mice, and those cells induced more osteolysis than cells not overexpressing GLI2 [78]. A similar study using siRNA to knockdown GLI2 expression showed the same results [79].

This overview of oncogenic activities of GLI2 in cancer highlights the many mechanisms by which GLI2 can modulate cell function. The breadth of GLI2 involvement in cell proliferation, survival, invasiveness, and modulation of the tumor microenvironment makes it an attractive target for anticancer therapeutics. However, much more is needed to be understood regarding the oncogenic tendencies of GLI2 as well as the ways in which GLI2 itself is regulated in order to further potential targeting strategies.

\section{Mechanisms Regulating GLI2 Activity}

One fascinating aspect of GLI2 is that its gene expression and protein activity can be modulated by a number of signaling pathways. This allows it to function as a mediator of crosstalk between these pathways (fig. 3). As many of the signaling mechanisms that can act on GLI2 are important for cell growth, they also help give importance to the role GLI2 plays in mediating all biological functions that have been outlined. Much of the background information regarding the protein domains of GLI2 along with important posttranslational modifications lends importance to the ways in which GLI2 activity can be manipulated by these various signaling pathways. The following section is dedicated to highlighting the different ways in which GLI2 expression is regulated.

\section{Canonical Modulation of GLI2 Protein Activity}

The Hh pathway is often thought of as the canonical pathway through which GLI2 activity is regulated. In fact, most of the early studies of GLI2 were performed in an attempt to understand how Hh signaling regulates development. As more became known about the function of GLI2, it was observed that GLI2 resulted in cellular effects independent of active $\mathrm{Hh}$ signaling. Nonetheless, GLI2 is critical in directing Hh signaling, and how this occurs has been explained in detail.

The canonical signaling through the Hh pathway consists of a 12-pass transmembrane receptor called Patched (PTCH) that exerts a consistent repressive effect on the 7-pass transmembrane receptor Smoothened (SMO) when the Hh ligand is absent. When the Hh ligand is present and binds to $\mathrm{PTCH}$, repression over SMO is released. This results in the transduction of the signal through a protein complex that includes SuFu, Kif7, and GLI. Generally, GLI2 is present in mammalian cells in its full-length form. The full-length GLI2 is bound to $\mathrm{SuFu}$, and thus SuFu keeps GLI2 tethered in the cytoplasm $[7,9,10]$. Another member of the pathway, Kif7, can antagonize SuFu by disrupting the SuFu/GLI2 complex and allowing nuclear translocation of GLI2 [80]. The absence of Hh signaling results in the predominance of GLI2 protein maintained in a repressive state while active Hh signaling reverses this repression. 
McCleary-Wheeler: From Normal Development to Disease: The Biochemistry and Regulation of GLI2

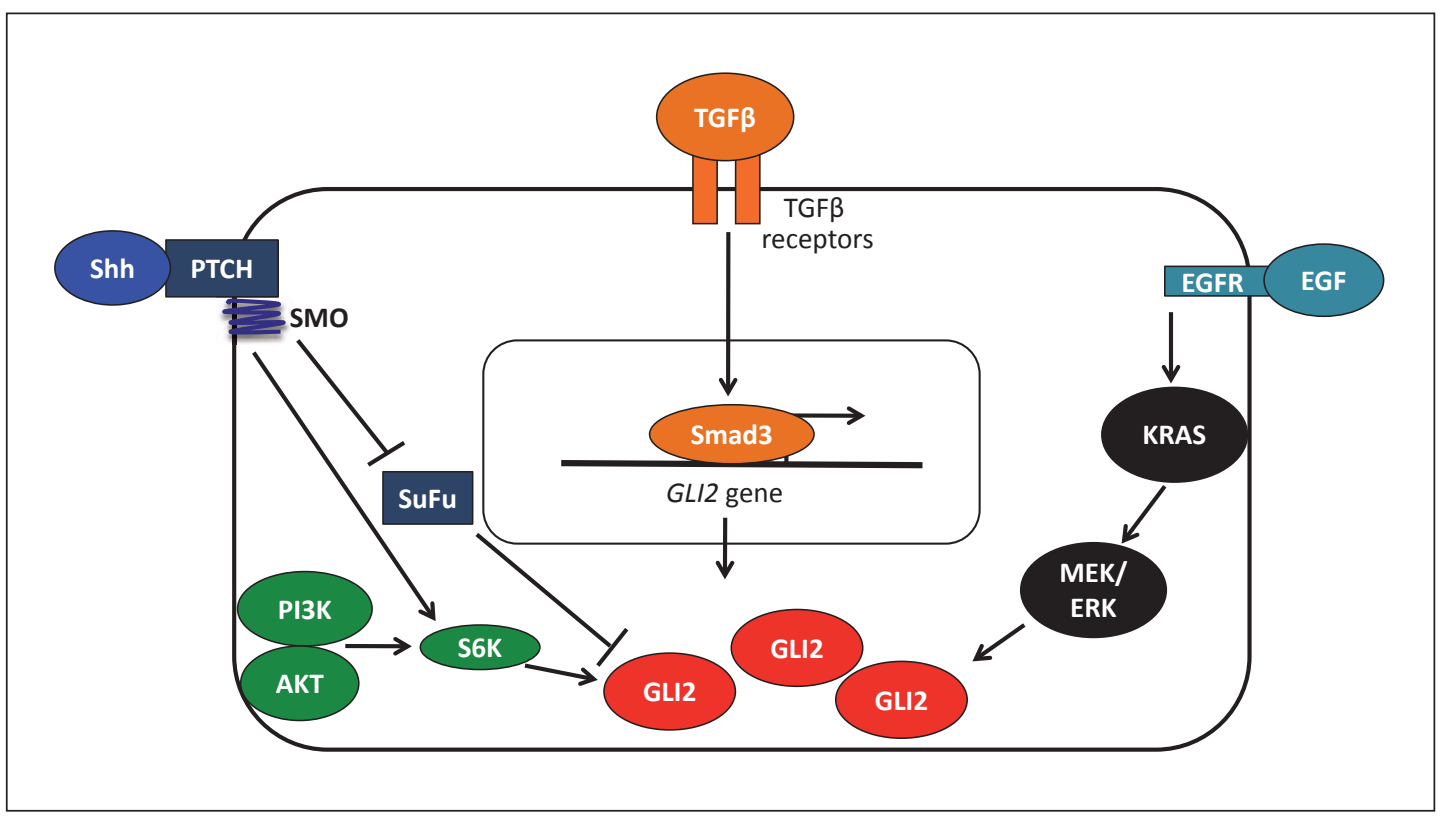

Fig. 3. Various signaling pathways are able to activate GLI2 at either the gene or protein levels. TGF $\beta$ signaling is able to directly increase GLI2 gene expression. Other signaling pathways, such as Hh, EGF, PI3K/AKT, and KRAS, are all able to act on the GLI2 protein to stabilize it and increase its activity.

What is most interesting is that the GLI1, and to some extent the GLI3 genes, are themselves targets of Hh signaling. Activation of the Hh pathway results in their transcriptional upregulation by the action of GLI2. However, the GLI2 gene is not activated by Hh signaling. While GLI1 is thought of as the potent activating transcription factor of the Hh pathway, it cannot serve in that role without its own transcriptional activation by GLI2. Thus, GLI2 is vital to Hh signaling, and this was highlighted in its role in development. This immediately identifies GLI2 as a unique effector of the Hh pathway and leaves in question how else GLI2 may be regulated.

\section{Noncanonical Regulation of the GLI2 Protein}

GLI2 is a point of crosstalk between signaling mechanisms. Multiple signaling mechanisms exist through which GLI2 expression can be manipulated aside from the canonical Hh pathway. One principle mechanism of altering the activity of the GLI2 protein is through posttranslational modifications, such as phosphorylation. Signaling cascades that are capable of this may have downstream components that modulate GLI2 in either an Hh-dependent manner or in a manner independent of Hh signaling. Several noncanonical signaling pathways that include PI3K/AKT, epidermal growth factor (EGF), and KRAS are capable of altering the GLI2 protein function.

It has been demonstrated that PI3K and AKT signaling are needed for Hh-mediated signaling. Upon the binding of Shh to PTCH, inhibition of SMO is relieved and simultaneously PI3K and AKT are activated [81]. PI3K and AKT antagonize PKA-mediated phosphorylation of GLI2 and subsequent stabilization of the activator form of GLI2 [81]. More detailed insights into the cooperation between $\mathrm{Hh}$ and PI3K/AKT pathways was elucidated in glioblastomas. In this tumor model, the PTEN gene is often lost, and this results in activation of AKT and Hh. It was found that AKT and Hh intersected at the level of S6K [82]. S6K, an effector of the mTOR complex downstream of AKT, is further stimulated by SMO [82]. Then, the synergistic acti- 
vation of S6K by the two pathways enhanced the GLI-dependent transcription [82]. Thus, it is evident from this overlap that activation of PI3K or AKT alone may be sufficient to stabilize GLI2 and result in upregulation of GLI2 activity in a manner that is independent of Hh signaling. Indeed PI3K/AKT can act independently of Hh. Other studies found that active ERK signaling works synergistically with Hh to regulate GLI protein activity [83]. Upon treatment of NIH $3 \mathrm{~T} 3$ cells with phorbol esters or with basic fibroblastic growth factor to activate ERK, GLI2 activity increased, and target gene expression likewise increased [83]. This finding was determined to be due to the activity of protein kinase $C-\delta$ and mitogen-activated protein/extracellular signal-regulated kinase-1 (MEK-1), which are able to activate both basal and overexpressed GLI2 proteins [83]. While physical interactions of these proteins with GLI2 were not directly evaluated, it was found that MEK-1 interacts with GLI1 at its N-terminus (amino acids 1-130), and this domain of GLI1 is required for increased activity [83]. It is reasonable that the effect exerted over GLI2 likely involves a similar interaction. While more mechanistic detail in this process has not been established, it may be that this interaction directs GLI subcellular localization and hence contributes to the increase in the observed activity.

Similar to the AKT/PI3K signaling, EGF signaling also activates GLI2 through a couple of different mechanisms. EGF through RAS/RAF/MEK/ERK signaling stabilizes GLI2 and prevents its degradation by the proteosome, thus increasing the GLI2 activity [84]. Another related study detailed a synergistic action of EGF and Hh on GLI2 wherein EGF activation and signal transduction through RAS/RAF/MEK/ERK stimulates JUN/AP-1 binding to DNA as well as JUN/AP-1 cooperation with activator forms of GLI to direct dual-pathway activation of target genes [85]. JUN expression increased with augmenting GLI activity, and JUN contains GLI-binding sites in its promoter, suggesting that this resultant positive feedback amplifies the target gene expression $[85,86]$. In vitro evaluation of mouse basal cell carcinoma cells treated with an EGF receptor inhibitor, gefitinib, and the GLI inhibitor GANT1 decreased cellular proliferation and survival greater than either inhibitor used as a single agent [85]. In a manner independent of EGF, KRAS signaling activates GLI activity.

Activated KRAS signaling also increases GLI2 activity independent of the Hh status of the cell. This phenomenon is well documented in pancreatic cancer with GLI1, although the exact mechanism of this relationship is still under investigation. Several observations have been made in understanding the noncanonical regulation of GLI2 in pancreatic ductal adenocarcinoma (PDAC). A transgenic mouse conditionally expressing a dominant-active form of Gli2 mainly in the pancreatic epithelium was developed that allowed insight specifically into the role of GLI2 in PDAC formation [63]. The tumors that subsequently formed in the pancreas were undifferentiated and did not recapitulate human PDAC histologically [63]. However, when these mice were crossed with $\mathrm{Kras}^{G 12 D}$ mutant mice, preneoplastic lesions formed and progression to invasive carcinoma occurred earlier [63]. These results indicate that while Gli2 activation alone does not mimic the progression of human PDAC, its dysfunction along with the initial Kras mutation do result in stages of disease that model human PDAC. Furthermore, the $\mathrm{Kras}^{G 12 D}$ mutation induces loss of primary cilia in PDAC precursor lesions and PDAC cells, and loss of primary cilia has been suggested to increase GLI2 activity [50,87]. Several studies have shown the cooperative integration of Gli1 and Kras in mouse models of PDAC [88-90], but the question remains where and how alterations and GLI2 activity affect this outcome. Nolan-Stevaux et al. [88] evaluated the role of Kras in GLI regulation by eliminating Hh activation in a Kras/p53 mouse model of PDAC. GLI1 expression remained high despite the loss of functional Hh signaling. Furthermore, in cell lines, depletion of Kras with siRNA resulted in a downregulation of Gli1, and loss of Gli1 results in apoptosis in these cells [88]. Additional in vivo evidence linking KRAS signaling with GLI1 was provided through the use of the Kras ${ }^{G 12 D}$ transgenic mouse crossed with total GLI1 knockout mice. These mice developed no tumors and fewer precursor lesions in the pancreas than those mice with only 
the $\operatorname{Kras}^{G 12 D}$ mutation and wild type for Gli1 [90]. Gli1 was found to mediate the oncogenic effects of the mutant Kras through modulation of an IL-6/STAT3 pathway functioning between the tumor cells and the tumor microenvironment [90]. While these extensive studies have focused on the interaction between KRAS and GLI1, the similarity between GLI1 and GLI2 and data with GLI2 alone suggest that a similar mechanism may exist between KRAS and GLI2. However, further studies are needed to elucidate the mechanisms of interactions between KRAS and GLI2 as well as GLI1.

All the above-described pathways highlight that GLI2 serves as a point of convergence for several signaling pathways. They can function together to act in a synergistic manner or independently to control GLI2. Whether GLI2 is activated in a canonical manner through $\mathrm{Hh}$ signaling or in a noncanonical manner through the action of other pathways, one point of GLI2 regulation has not been mentioned. How is GLI2 gene regulation controlled? While some answers to this question have been found, it is still largely unknown and presents an important area for discovery.

\section{Transcriptional Activation of GLI2 Expression}

While the above examples outline overlapping interactions of signaling pathways through GLI2, the mechanisms involve modulation of the GLI2 protein. Whether through alterations in protein stability or subcellular localization, these changes affect the activity of GLI2 at the protein level. A significant question remains regarding how the GLI2 gene is regulated. An interesting observation was made using a genome-wide analysis of human PDAC samples and found mutations in several Hh pathway members with the exception of GLI2 [91]. Yet, overexpression of GLI2 in tumors has been well described. Only a few mechanisms of GLI2 gene regulation have been documented, and only a few transcription factors are known that directly regulated GLI2 gene expression.

Perhaps one of the most well-characterized signaling pathways known to alter GLI2 gene expression is that of TGF $\beta$. The TGF $\beta$ pathway can directly and rapidly induce transcription of GLI2. This was demonstrated in numerous cell lines as well as in transgenic mice where TGF $\beta 1$ is overexpressed in the skin $[92,93]$. This work showed that this phenomenon occurs through the binding of Smad 3 to a Smad-binding element located $30 \mathrm{bp}$ upstream of the transcription start site of GLI2 [93]. The TGFß-responsive element in the GLI2 promoter was mapped to an area located between 29 and 119 bp upstream of the transcription start site [93]. This not only includes the Smad3-binding element, but also a TCF/LEF-binding site whereby Wnt signaling can cooperate through the action of $\beta$-catenin binding to this site [93]. However, other considerations, such as the recruitment of transcriptional coactivators or how the composition of the chromatin landscape of this region of the GLI2 promoter changes, have not been investigated.

Aside from TGF $\beta$, other pathways can regulate GLI2 gene expression. The cytokine CCL5 can act in stromal cells through the PI3K-AKT-NFKB pathway to stimulate the transcriptional activation of GLI2 [77]. Another mode of GLI2 transcriptional activation is through Atoh1. Atoh1 is a transcription factor that is highly expressed in GNP cells, the cell population that gives rise to medulloblastomas [94]. Medulloblastomas that have a high expression level of Atoh1 are associated with a poorer prognosis [95]. Utilizing Atoh $1^{\text {flox/flox }}$ mice, GNP cells were isolated from the cerebellum of 5-day-old postnatal mice and transduced with adenoviruses having either green fluorescent protein or Cre recombinase genes. In the cells lacking Atoh1, proliferation was decreased along with Gli2 expression [96]. This led to further investigations into the mechanisms of Atoh1 downregulation of Gli2. Atoh1 activates target genes through binding to E-boxes. Flora et al. [96] identified an eight E-box sequence stretch in the second intron of mouse Gli2 and looked for conservation with human GLI2. Two of these E-boxes were conserved in mouse and human and both EMSA and ChIP analysis showed binding of 
Atoh1 to this region of Gli2 [96]. They further confirmed that this was suggestive of a transcriptional mechanism acting through an enhancer element, as the presence of monomethylated lysine 4 of histone H3 was identified via ChIP analysis. This mechanism of GLI2 gene regulation by Atoh1 is involved in differentiation of neurons, secretory cells of the gastrointestinal tract, and some mechanoreceptors [97].

\section{Conclusions}

GLI2 is a critical gene responsible for regulating normal cellular functions and particularly normal development. Under most circumstances, GLI2 mediates its effects as a transcriptional activator of its target genes, but in some cases, it functions as a transcriptional repressor. The various domains constituting this protein contribute to the variety of mechanisms that are able to regulate its activity, making GLI2 a point of convergence of several signaling pathways. This idea is amplified in the context of the role of GLI2 in disease. While loss-of-function of GLI2 through gene mutations can result in failure of normal embryonic development and the formation of congenital anomalies, mutations in the GLI2 gene are rarely identified as a cause of spontaneous disease arising in mature organisms. Instead, it appears that the gain-of-function of GLI2 precipitating its oncogenic functions is a result of misregulation of the protein or gene transcription that results in its constitutive activation or overexpression. Although some mechanisms of GLI2 gene regulation have become known and been well defined, little is currently known about its regulation at the transcriptional and chromatin levels. Given the range of biologic implications, particularly concerning its oncogenic potential, these points of GLI2 regulation must be further explored to fully understand the role GLI2 has in disease. With increased knowledge, the mechanisms of GLI2 regulation could be potentially exploited for therapeutic use. While targeting GLI2 for therapeutic means may be attractive, consideration must be given to the complexity of pathways regulating GLI2. As such, the targeting of one pathway in order to alter GLI2 activity may be insufficient to elicit a biologically relevant response in cancers. Furthering our understanding of the most downstream mechanisms of GLI2 regulation will likely be a critical strategy to overcoming the challenges associated with targeting it.

\section{References}

1 Pavletich NP, Pabo CO: Crystal structure of a five-finger GLI-DNA complex: new perspectives on zinc fingers. Science 1993;261:1701-1707.

2 Ruppert JM, Kinzler KW, Wong AJ, Bigner SH, Kao FT, Law ML, Seuanez HN, O’Brien SJ, Vogelstein B: The GLIKruppel family of human genes. Mol Cell Biol 1988;8:3104-3113.

3 Kinzler KW, Vogelstein B: The GLI gene encodes a nuclear protein which binds specific sequences in the human genome. Mol Cell Biol 1990;10:634-642.

4 Kinzler KW, Ruppert JM, Bigner SH, Vogelstein B: The GLI gene is a member of the Kruppel family of zinc finger proteins. Nature 1988;332:371-374.

5 Dai P, Akimaru H, Tanaka Y, Maekawa T, Nakafuku M, Ishii S: Sonic hedgehog-induced activation of the GLI1 promoter is mediated by GLI3. J Biol Chem 1999;274:8143-8152.

6 Sasaki H, Nishizaki Y, Hui C, Nakafuku M, Kondoh H: Regulation of Gli2 and Gli3 activities by an amino-terminal repression domain: implication of Gli2 and Gli3 as primary mediators of Shh signaling. Development 1999; 126:3915-3924.

7 Dunaeva M, Michelson P, Kogerman P, Toftgard R: Characterization of the physical interaction of Gli proteins with SUFU proteins. J Biol Chem 2003;278:5116-5122.

8 Hatayama M, Aruga J: Gli protein nuclear localization signal. Vitam Horm 2012;88:73-89.

9 Ding Q, Fukami S, Meng X, Nishizaki Y, Zhang X, Sasaki H, Dlugosz A, Nakafuku M, Hui C: Mouse suppressor of fused is a negative regulator of sonic hedgehog signaling and alters the subcellular distribution of Gli1. Curr Biol 1999;9:1119-1122. 
10 Merchant M, Vajdos FF, Ultsch M, Maun HR, Wendt U, Cannon J, Desmarais W, Lazarus RA, de Vos AM, de Sauvage FJ: Suppressor of fused regulates Gli activity through a dual binding mechanism. Mol Cell Biol 2004; 24:8627-8641.

11 Kang S, Graham JM Jr, Olney AH, Biesecker LG: Gli3 frameshift mutations cause autosomal dominant pallisterhall syndrome. Nat Genet 1997;15:266-268.

12 Canettieri G, Di Marcotullio L, Greco A, Coni S, Antonucci L, Infante P, Pietrosanti L, De Smaele E, Ferretti E, Miele E, Pelloni M, De Simone G, Pedone EM, Gallinari P, Giorgi A, Steinkuhler C, Vitagliano L, Pedone C, Schinin ME, Screpanti I, Gulino A: Histone deacetylase and cullin3-ren(kctd11) ubiquitin ligase interplay regulates hedgehog signalling through Gli acetylation. Nat Cell Biol 2010;12:132-142.

13 Coni S, Antonucci L, D’Amico D, Di Magno L, Infante P, De Smaele E, Giannini G, Di Marcotullio L, Screpanti I, Gulino A, Canettieri G: Gli2 acetylation at lysine 757 regulates hedgehog-dependent transcriptional output by preventing its promoter occupancy. PLoS One 2013;8:e65718.

14 Cheng SY, Bishop JM: Suppressor of Fused represses Gli-mediated transcription by recruiting the SAP18mSin3 corepressor complex. Proc Natl Acad Sci USA 2002;99:5442-5447.

15 Akimaru H, Chen Y, Dai P, Hou DX, Nonaka M, Smolik SM, Armstrong S, Goodman RH, Ishii S: Drosophila CBP is a co-activator of cubitus interruptus in hedgehog signalling. Nature 1997;386:735-738.

16 Hughes DC, Allen J, Morley G, Sutherland K, Ahmed W, Prosser J, Lettice L, Allan G, Mattei MG, Farrall M, Hill RE: Cloning and sequencing of the mouse Gli2 gene: localization to the dominant hemimelia critical region. Genomics 1997;39:205-215.

17 Han L, Pan Y, Wang B: Small ubiquitin-like Modifier (SUMO) modification inhibits GLI2 protein transcriptional activity in vitro and in vivo. J Biol Chem 2012;287:20483-20489.

18 Hui CC, Slusarski D, Platt KA, Holmgren R, Joyner AL: Expression of three mouse homologs of the drosophila segment polarity gene cubitus interruptus, Gli, Gli-2, and Gli-3, in ectoderm- and mesoderm-derived tissues suggests multiple roles during postimplantation development. Dev Biol 1994;162:402-413.

19 Vortkamp A, Gessler M, Grzeschik KH: GLI3 zinc-finger gene interrupted by translocations in Greig syndrome families. Nature 1991;352:539-540.

20 Hui CC, Joyner AL: A mouse model of greig cephalopolysyndactyly syndrome: the extra-toesJ mutation contains an intragenic deletion of the Gli3 gene. Nat Genet 1993;3:241-246.

21 Radhakrishna U, Wild A, Grzeschik KH, Antonarakis SE: Mutation in GLI3 in postaxial polydactyly type A. Nat Genet 1997;17:269-271.

22 Mo R, Freer AM, Zinyk DL, Crackower MA, Michaud J, Heng HH, Chik KW, Shi XM, Tsui LC, Cheng SH, Joyner AL, Hui C: Specific and redundant functions of Gli2 and Gli3 zinc finger genes in skeletal patterning and development. Development 1997;124:113-123.

23 Motoyama J, Liu J, Mo R, Ding Q, Post M, Hui CC: Essential function of Gli2 and Gli3 in the formation of lung, trachea and oesophagus. Nat Genet 1998;20:54-57.

24 Park HL, Bai C, Platt KA, Matise MP, Beeghly A, Hui CC, Nakashima M, Joyner AL: Mouse Gli1 mutants are viable but have defects in Shh signaling in combination with a Gli2 mutation. Development 2000;127:1593-1605.

25 Wang Y, Martin JF, Bai CB: Direct and indirect requirements of Shh/Gli signaling in early pituitary development. Dev Biol 2010;348:199-209.

26 Matise MP, Epstein DJ, Park HL, Platt KA, Joyner AL: Gli2 is required for induction of floor plate and adjacent cells, but not most ventral neurons in the mouse central nervous system. Development 1998;125:2759-2770.

27 Ding Q, Motoyama J, Gasca S, Mo R, Sasaki H, Rossant J, Hui CC: Diminished sonic hedgehog signaling and lack of floor plate differentiation in Gli2 mutant mice. Development 1998;125:2533-2543.

28 Bai CB, Joyner AL: Gli1 can rescue the in vivo function of Gli2. Development 2001;128:5161-5172.

29 Ingham PW, McMahon AP: Hedgehog signaling in animal development: paradigms and principles. Genes Dev 2001;15:3059-3087.

30 Qi Y, Tan M, Hui CC, Qiu M: Gli2 is required for normal Shh signaling and oligodendrocyte development in the spinal cord. Mol Cell Neurosci 2003;23:440-450.

31 Motoyama J, Milenkovic L, Iwama M, Shikata Y, Scott MP, Hui CC: Differential requirement for Gli2 and Gli3 in ventral neural cell fate specification. Dev Biol 2003;259:150-161.

32 Brewster R, Mullor JL, Ruiz i Altaba A: Gli2 functions in FGF signaling during antero-posterior patterning. Development 2000;127:4395-4405.

33 Blaess S, Corrales JD, Joyner AL: Sonic hedgehog regulates Gli activator and repressor functions with spatial and temporal precision in the mid/hindbrain region. Development 2006;133:1799-1809.

34 Nguyen V, Chokas AL, Stecca B, Ruiz i Altaba A: Cooperative requirement of the Gli proteins in neurogenesis. Development 2005;132:3267-3279.

35 Po A, Ferretti E, Miele E, De Smaele E, Paganelli A, Canettieri G, Coni S, Di Marcotullio L, Biffoni M, Massimi L, Di Rocco C, Screpanti I, Gulino A: Hedgehog controls neural stem cells through p53-independent regulation of Nanog. EMBO J 2010;29:2646-2658.

36 Takanaga H, Tsuchida-Straeten N, Nishide K, Watanabe A, Aburatani H, Kondo T: Gli2 is a novel regulator of sox2 expression in telencephalic neuroepithelial cells. Stem Cells 2009;27:165-174.

37 Voronova A, Fischer A, Ryan T, Al Madhoun A, Skerjanc IS: Ascl1/Mash1 is a novel target of Gli2 during Gli2induced neurogenesis in P19 EC cells. PLoS One 2011;6:e19174.

38 Corrales JD, Rocco GL, Blaess S, Guo Q, Joyner AL: Spatial pattern of sonic hedgehog signaling through Gli genes during cerebellum development. Development 2004;131:5581-5590. 
39 Corrales JD, Blaess S, Mahoney EM, Joyner AL: The level of sonic hedgehog signaling regulates the complexity of cerebellar foliation. Development 2006;133:1811-1821.

40 Bok J, Dolson DK, Hill P, Ruther U, Epstein DJ, Wu DK: Opposing gradients of Gli repressor and activators mediate Shh signaling along the dorsoventral axis of the inner ear. Development 2007;134:1713-1722.

41 Bowers M, Eng L, Lao Z, Turnbull RK, Bao X, Riedel E, Mackem S, Joyner AL: Limb anterior-posterior polarity integrates activator and repressor functions of Gli2 as well as Gli3. Dev Biol 2012;370:110-124.

42 Buttitta L, Mo R, Hui CC, Fan CM: Interplays of Gli2 and Gli3 and their requirement in mediating Shh-dependent sclerotome induction. Development 2003;130:6233-6243.

43 Shimoyama A, Wada M, Ikeda F, Hata K, Matsubara T, Nifuji A, Noda M, Amano K, Yamaguchi A, Nishimura R, Yoneda T: Ihh/Gli2 signaling promotes osteoblast differentiation by regulating Runx2 expression and function. Mol Biol Cell 2007;18:2411-2418.

44 Zhao M, Qiao M, Harris SE, Chen D, Oyajobi BO, Mundy GR: The zinc finger transcription factor Gli2 mediates bone morphogenetic protein 2 expression in osteoblasts in response to hedgehog signaling. Mol Cell Biol 2006;26:6197-6208.

45 Mill P, Mo R, Fu H, Grachtchouk M, Kim PC, Dlugosz AA, Hui CC: Sonic hedgehog-dependent activation of Gli2 is essential for embryonic hair follicle development. Genes Dev 2003;17:282-294.

46 Rutter M, Wang J, Huang Z, Kuliszewski M, Post M: Gli2 influences proliferation in the developing lung through regulation of cyclin expression. Am J Respir Cell Mol Biol 2010;42:615-625.

47 Rowbotham NJ, Hager-Theodorides AL, Furmanski AL, Ross SE, Outram SV, Dessens JT, Crompton T: Sonic hedgehog negatively regulates pre-TCR-induced differentiation by a Gli2-dependent mechanism. Blood 2009; 113:5144-5156.

48 Voronova A, Al Madhoun A, Fischer A, Shelton M, Karamboulas C, Skerjanc IS: Gli2 and MEF2C activate each other's expression and function synergistically during cardiomyogenesis in vitro. Nucleic Acids Res 2012;40: 3329-3347.

49 McDermott A, Gustafsson M, Elsam T, Hui CC, Emerson CP Jr, Borycki AG: Gli2 and Gli3 have redundant and context-dependent function in skeletal muscle formation. Development 2005;132:345-357.

50 Cervantes S, Lau J, Cano DA, Borromeo-Austin C, Hebrok M: Primary cilia regulate Gli/hedgehog activation in pancreas. Proc Natl Acad Sci USA 2010;107:10109-10114.

51 Roessler E, Du YZ, Mullor JL, Casas E, Allen WP, Gillessen-Kaesbach G, Roeder ER, Ming JE, Ruiz i Altaba A, Muenke M: Loss-of-function mutations in the human Gli2 gene are associated with pituitary anomalies and holoprosencephaly-like features. Proc Natl Acad Sci USA 2003;100:13424-13429.

52 Franca MM, Jorge AA, Carvalho LR, Costalonga EF, Vasques GA, Leite CC, Mendonca BB, Arnhold IJ: Novel heterozygous nonsense Gli2 mutations in patients with hypopituitarism and ectopic posterior pituitary lobe without holoprosencephaly. J Clin Endocrinol Metab 2010;95:E384-E391.

53 Bertolacini CD, Ribeiro-Bicudo LA, Petrin A, Richieri-Costa A, Murray JC: Clinical findings in patients with Gli2 mutations-phenotypic variability. Clin Genet 2012;81:70-75.

54 Kinzler KW, Bigner SH, Bigner DD, Trent JM, Law ML, O’Brien SJ, Wong AJ, Vogelstein B: Identification of an amplified, highly expressed gene in a human glioma. Science 1987;236:70-73.

55 Grachtchouk M, Mo R, Yu S, Zhang X, Sasaki H, Hui CC, Dlugosz AA: Basal cell carcinomas in mice overexpressing Gli2 in skin. Nat Genet 2000;24:216-217.

56 Kim JE, Singh RR, Cho-Vega JH, Drakos E, Davuluri Y, Khokhar FA, Fayad L, Medeiros LJ, Vega F: Sonic hedgehog signaling proteins and ATP-binding cassette G2 are aberrantly expressed in diffuse large B-cell lymphoma. Mod Pathol 2009;22:1312-1320.

57 Kim KH, Kim JM, Choi YL, Shin YK, Lee HC, Seong IO, Kim BK, Chae SW, Chung YS, Kim SH: Expression of sonic hedgehog signaling molecules in normal, hyperplastic and carcinomatous endometrium. Pathol Int 2009;59: 279-287.

58 Yang W, Liu X, Choy E, Mankin H, Hornicek FJ, Duan Z: Targeting hedgehog-GLI-2 pathway in osteosarcoma. J Orthop Res 2013;31:502-509.

59 Alexaki VI, Javelaud D, Van Kempen LC, Mohammad KS, Dennler S, Luciani F, Hoek KS, Juarez P, Goydos JS, Fournier PJ, Sibon C, Bertolotto C, Verrecchia F, Saule S, Delmas V, Ballotti R, Larue L, Saiag P, Guise TA, Mauviel A: Gli2-mediated melanoma invasion and metastasis. J Natl Cancer Inst 2010;102:1148-1159.

60 Mazumdar T, DeVecchio J, Shi T, Jones J, Agyeman A, Houghton JA: Hedgehog signaling drives cellular survival in human colon carcinoma cells. Cancer Res 2011;71:1092-1102.

61 Narita S, So A, Ettinger S, Hayashi N, Muramaki M, Fazli L, Kim Y, Gleave ME: Gli2 knockdown using an antisense oligonucleotide induces apoptosis and chemosensitizes cells to paclitaxel in androgen-independent prostate cancer. Clin Cancer Res 2008;14:5769-5777.

62 Mechlin CW, Tanner MJ, Chen M, Buttyan R, Levin RM, Mian BM: Gli2 expression and human bladder transitional carcinoma cell invasiveness. J Urol 2010;184:344-351.

63 Pasca di Magliano M, Sekine S, Ermilov A, Ferris J, Dlugosz AA, Hebrok M: Hedgehog/Ras interactions regulate early stages of pancreatic cancer. Genes Dev 2006;20:3161-3173.

64 Hu M, Yao J, Carroll DK, Weremowicz S, Chen H, Carrasco D, Richardson A, Violette S, Nikolskaya T, Nikolsky Y, Bauerlein EL, Hahn WC, Gelman RS, Allred C, Bissell MJ, Schnitt S, Polyak K: Regulation of in situ to invasive breast carcinoma transition. Cancer Cell 2008;13:394-406. 
65 Edson MA, Nalam RL, Clementi C, Franco HL, Demayo FJ, Lyons KM, Pangas SA, Matzuk MM: Granulosa cellexpressed BMPR1a and BMPR1b have unique functions in regulating fertility but act redundantly to suppress ovarian tumor development. Mol Endocrinol 2010;24:1251-1266.

66 Hutchin ME, Kariapper MS, Grachtchouk M, Wang A, Wei L, Cummings D, Liu J, Michael LE, Glick A, Dlugosz AA: Sustained hedgehog signaling is required for basal cell carcinoma proliferation and survival: conditional skin tumorigenesis recapitulates the hair growth cycle. Genes Dev 2005;19:214-223.

67 Thiyagarajan S, Bhatia N, Reagan-Shaw S, Cozma D, Thomas-Tikhonenko A, Ahmad N, Spiegelman VS: Role of Gli2 transcription factor in growth and tumorigenicity of prostate cells. Cancer Res 2007;67:10642-10646.

68 Eichberger T, Sander V, Schnidar H, Regl G, Kasper M, Schmid C, Plamberger S, Kaser A, Aberger F, Frischauf AM: Overlapping and distinct transcriptional regulator properties of the Gli1 and Gli2 oncogenes. Genomics 2006;87:616-632.

69 Regl G, Kasper M, Schnidar H, Eichberger T, Neill GW, Ikram MS, Quinn AG, Philpott MP, Frischauf AM, Aberger F: The zinc-finger transcription factor Gli2 antagonizes contact inhibition and differentiation of human epidermal cells. Oncogene 2004;23:1263-1274.

70 Bishop CL, Bergin AM, Fessart D, Borgdorff V, Hatzimasoura E, Garbe JC, Stampfer MR, Koh J, Beach DH: Primary cilium-dependent and -independent hedgehog signaling inhibits p16(ink4a). Mol Cell 2010;40:533547.

71 Kim Y, Yoon JW, Xiao X, Dean NM, Monia BP, Marcusson EG: Selective down-regulation of glioma-associated oncogene 2 inhibits the proliferation of hepatocellular carcinoma cells. Cancer Res 2007;67:3583-3593.

72 Kump E, Ji J, Wernli M, Hausermann P, Erb P: Gli2 upregulates cFlip and renders basal cell carcinoma cells resistant to death ligand-mediated apoptosis. Oncogene 2008;27:3856-3864.

73 Singh BN, Fu J, Srivastava RK, Shankar S: Hedgehog signaling antagonist GDC-0449 (Vismodegib) inhibits pancreatic cancer stem cell characteristics: molecular mechanisms. PLoS One 2011;6:e27306.

74 Zhang D, Cao L, Li Y, Lu H, Yang X, Xue P: Expression of glioma-associated oncogene 2 (Gli 2) is correlated with poor prognosis in patients with hepatocellular carcinoma undergoing hepatectomy. World J Surg Oncol 2013; 11:25.

75 Perrot CY, Javelaud D, Mauviel A: Overlapping activities of TGF-beta and Hedgehog signaling in cancer: therapeutic targets for cancer treatment. Pharmacol Ther 2013;137:183-199.

76 Furler RL, Uittenbogaart CH: GLI2 regulates TGF-beta1 in human CD4+ T cells: implications in cancer and HIV pathogenesis. PLoS One 2012; 7:e40874.

77 Elsawa SF, Almada LL, Ziesmer SC, Novak AJ, Witzig TE, Ansell SM, Fernandez-Zapico ME: Gli2 transcription factor mediates cytokine cross-talk in the tumor microenvironment. J Biol Chem 2011;286:21524-21534.

78 Sterling JA, Oyajobi BO, Grubbs B, Padalecki SS, Munoz SA, Gupta A, Story B, Zhao M, Mundy GR: The hedgehog signaling molecule Gli2 induces parathyroid hormone-related peptide expression and osteolysis in metastatic human breast cancer cells. Cancer Res 2006;66:7548-7553.

79 Johnson RW, Nguyen MP, Padalecki SS, Grubbs BG, Merkel AR, Oyajobi BO, Matrisian LM, Mundy GR, Sterling JA: TGF-beta promotion of Gli2-induced expression of parathyroid hormone-related protein, an important osteolytic factor in bone metastasis, is independent of canonical hedgehog signaling. Cancer Res 2011;71: 822-831.

80 Li ZJ, Nieuwenhuis E, Nien W, Zhang X, Zhang J, Puviindran V, Wainwright BJ, Kim PC, Hui CC: Kif7 regulates Gli2 through Sufu-dependent and -independent functions during skin development and tumorigenesis. Development 2012;139:4152-4161.

81 Riobo NA, Lu K, Ai X, Haines GM, Emerson CP Jr: Phosphoinositide 3-kinase and Akt are essential for sonic hedgehog signaling. Proc Natl Acad Sci USA 2006;103:4505-4510.

82 Gruber Filbin M, Dabral SK, Pazyra-Murphy MF, Ramkissoon S, Kung AL, Pak E, Chung J, Theisen MA, Sun Y, Franchetti Y, Shulman DS, Redjal N, Tabak B, Beroukhim R, Wang Q, Zhao J, Dorsch M, Buonamici S, Ligon KL, Kelleher JF, Segal RA: Coordinate activation of Shh and PI3K signaling in PTEN-deficient glioblastoma: new therapeutic opportunities. Nat Med 2013;19:1518-1523.

83 Riobo NA, Haines GM, Emerson CP Jr: Protein kinase C-delta and mitogen-activated protein/extracellular signal-regulated kinase-1 control GLI activation in hedgehog signaling. Cancer Res 2006;66:839-845.

84 Kasper M, Schnidar H, Neill GW, Hanneder M, Klingler S, Blaas L, Schmid C, Hauser-Kronberger C, Regl G, Philpott MP, Aberger F: Selective modulation of hedgehog/Gli target gene expression by epidermal growth factor signaling in human keratinocytes. Mol Cell Biol 2006;26:6283-6298.

85 Schnidar H, Eberl M, Klingler S, Mangelberger D, Kasper M, Hauser-Kronberger C, Regl G, Kroismayr R, Moriggl R, Sibilia M, Aberger F: Epidermal growth factor receptor signaling synergizes with Hedgehog/GLI in oncogenic transformation via activation of the MEK/ERK/JUN pathway. Cancer Res 2009;69:1284-1292.

86 Laner-Plamberger S, Kaser A, Paulischta M, Hauser-Kronberger C, Eichberger T, Frischauf AM: Cooperation between GLI and JUN enhances transcription of JUN and selected GLI target genes. Oncogene 2009;28:16391651.

87 Seeley ES, Carriere C, Goetze T, Longnecker DS, Korc M: Pancreatic cancer and precursor pancreatic intraepithelial neoplasia lesions are devoid of primary cilia. Cancer Res 2009;69:422-430.

88 Nolan-Stevaux O, Lau J, Truitt ML, Chu GC, Hebrok M, Fernandez-Zapico ME, Hanahan D: GLI1 is regulated through Smoothened-independent mechanisms in neoplastic pancreatic ducts and mediates PDAC cell survival and transformation. Genes Dev 2009;23:24-36. 
89 Stecca B, Mas C, Clement V, Zbinden M, Correa R, Piguet V, Beermann F, Ruiz IAA: Melanomas require hedgehogGLI signaling regulated by interactions between GLI1 and the RAS-MEK/AKT pathways. Proc Natl Acad Sci USA 2007;104:5895-5900.

90 Mills LD, Zhang Y, Marler RJ, Herreros-Villanueva M, Zhang L, Almada LL, Couch F, Wetmore C, Pasca di Magliano M, Fernandez-Zapico ME: Loss of the transcription factor GLI1 identifies a signaling network in the tumor microenvironment mediating KRAS oncogene-induced transformation. J Biol Chem 2013;288:1178611794.

91 Jones S, Zhang X, Parsons DW, Lin JC, Leary RJ, Angenendt P, Mankoo P, Carter H, Kamiyama H, Jimeno A, Hong SM, Fu B, Lin MT, Calhoun ES, Kamiyama M, Walter K, Nikolskaya T, Nikolsky Y, Hartigan J, Smith DR, Hidalgo M, Leach SD, Klein AP, Jaffee EM, Goggins M, Maitra A, Iacobuzio-Donahue C, Eshleman JR, Kern SE, Hruban RH, Karchin R, Papadopoulos N, Parmigiani G, Vogelstein B, Velculescu VE, Kinzler KW: Core signaling pathways in human pancreatic cancers revealed by global genomic analyses. Science 2008;321:1801-1806.

92 Dennler S, Andre J, Alexaki I, Li A, Magnaldo T, ten Dijke P, Wang XJ, Verrecchia F, Mauviel A: Induction of sonic hedgehog mediators by transforming growth factor-beta: Smad3-dependent activation of GLI2 and GLI1 expression in vitro and in vivo. Cancer Res 2007;67:6981-6986.

93 Dennler S, Andre J, Verrecchia F, Mauviel A: Cloning of the human GLI2 Promoter: transcriptional activation by transforming growth factor-beta via SMAD3/beta-catenin cooperation. J Biol Chem 2009;284:3152331531.

94 Bertrand N, Castro DS, Guillemot F: Proneural genes and the specification of neural cell types. Nat Rev Neurosci 2002;3:517-530.

95 Salsano E, Pollo B, Eoli M, Giordana MT, Finocchiaro G: Expression of MATH1, a marker of cerebellar granule cell progenitors, identifies different medulloblastoma sub-types. Neurosci Lett 2004;370:180-185.

96 Flora A, Klisch TJ, Schuster G, Zoghbi HY: Deletion of Atoh1 disrupts Sonic Hedgehog signaling in the developing cerebellum and prevents medulloblastoma. Science 2009;326:1424-1427.

97 Mulvaney J, Dabdoub A: Atoh1, an essential transcription factor in neurogenesis and intestinal and inner ear development: function, regulation, and context dependency. J Assoc Res Otolaryngol 2012;13:281-293. 